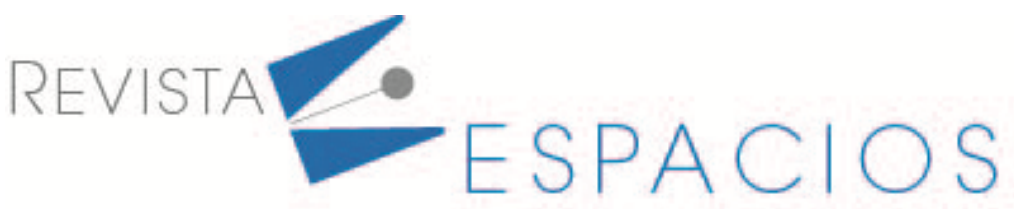

Vol. 41 (47) 2020 • Art. 1

Recibido/Received: 05/04/2020 • Aprobado/Approved: 20/11/2020 • Publicado/Published: 10/12/2020

\section{El embarazo adolescente como un problema de salud pública en Latinoamérica}

\section{Teen pregnancy as a public health problem in Latin America}

\author{
MARTINEZ, Enriqueta A. ${ }^{1}$ \\ MONTERO, Gloria I. ${ }^{2}$ \\ ZAMBRANO, Rosa M. ${ }^{3}$
}

\begin{abstract}
Resumen
El embarazo adolescente es una problemática de índole biológico, psicológico y social que involucra a la familia y a la sociedad. Esta investigación parte de un enfoque cualitativo con alcance descriptivo y exploratorio mediante el uso de revisiones de estudios realizados en Latinoamérica. Como resultado se destaca que en Latinoamérica el número de embarazos adolescentes es creciente, lo cual indica que algo está fallando en la educación sobre el manejo de la sexualidad a temprana edad.

Palabras clave: adolescencia, embarazo, problema biopsicosocial
\end{abstract}

\begin{abstract}
Teenage pregnancy is a biological, psychological and social problem that involves the family and society. This research starts from a qualitative approach with a descriptive and exploratory scope through the use of reviews of studies carried out in Latin America. As results, it is highlighted that in Latin America the number of adolescent pregnancies is increasing, which indicates something is missing in the education on the management of sexuality at an early age.
\end{abstract}

key words: adolescence, pregnancy, biopsychosocial problem

\section{Introducción}

El embarazo adolescente es un problema de salud que pone en riesgo tanto al niño como a la madre. La Organización Mundial de la Salud -OMS- (2018) menciona que la adolescencia está entendida entre los 10 y 19 años y se divide en adolescentes jóvenes (de 10 a 14 años) y segunda adolescencia (de 15 a 19 años). Las complicaciones tanto en el embarazo como en el parto, en la adolescencia, constituyen la primera causa de muerte en mujeres de 15 a 19 años a nivel mundial, por lo que Gaete (2015) refiere que se lo trata como una etapa de riesgo, ya que es un estado de cambio en el que aún no se ha alcanzado el desarrollo físico y emocional propio de la madurez. Para Pinzón, Ruiz, Aguilera, y Abril (2018) esta problemática se encuentra vinculada a

\footnotetext{
${ }^{1}$ Magíster en Ginecología Infanto Juvenil y Salud Reproductiva del Adolescente, Licenciada en Enfermería. Docente Enfermería. Universidad Católica de Cuenca.enriqueta.martinez@ucacue.edu.ec

${ }^{2}$ Magíster en Gerencia de Servicios de Salud, Licenciada en Enfermería. Docente Enfermería. Universidad Católica de Cuenca. gloria.montero@ucacue.edu.ec

${ }_{3}^{3}$ Doctorando, Magíster en Psicoanálisis con Mención en Educación, Sicólogo Clínico. Docente Enfermería. Universidad. rmzambranog@ucacue.edu.ec
} 
múltiples causas como un déficit educativo, escasez económica, hacinamiento, violencia dentro del hogar, desconocimiento del ciclo menstrual y anticoncepción, entre otras. Por lo que, embarazarse a corta edad además de tener relación con lo antes mencionado, en algunos casos, termina fortaleciéndolos al no permitir una proyección regular del desarrollo adolescente, truncando sus planes de vida y afectando no sólo la vida de la madre sino también la de su hijo.

Flores, Navas y Arenas (2017) establecen que "el embarazo en la adolescencia se considera un problema de salud pública por la Organización Mundial de la Salud (OMS) debido a las repercusiones biopsicosociales que tiene en la salud de la madre y del hijo(a)" (p.374). Una de ellas es la muerte de adolescentes durante el embarazo o en el parto, a esto se suma la falta de educación sexual y orientación familiar, todo lo cual conduce a incrementar la pobreza. Por su parte Loredo, Vargas, Casas, Gonzáles y Gutierrez (2017) mencionan que "de acuerdo con el Censo de Población y Vivienda realizado en México en el año 2005, el 17\% de los nacimientos corresponden a madres adolescentes" (p.224), estos índices estadísticos reflejan que el embarazo adolescente es un problema de salud pública que necesita ser enfrentado con propuestas desde diferentes actores.

Según Molina, Pena, Díaz, y Soto (2019) entre las causas de muerte de las adolescentes se encuentran las complicaciones del estado gestacional y el parto. Pero además tiene varias consecuencias negativas como aborto ilegal, abandono y maltrato de los niños, conflictos familiares, deserción escolar y múltiples problemas de salud tanto para el niño como para la madre.

Por su parte Morín, Treviño y Rivera (2019) indican que

De acuerdo con la Organización Mundial de la Salud los embarazos en la adolescencia tienen una grave repercusión en el ámbito biopsicosocial tanto de la madre como del hijo, además de que suelen producir una serie de complicaciones durante el embarazo y el parto, lo que se traduce en altas tasas de mortalidad (p.4).

Fawed, Erazo, Carrasco, Gonzáles, Mendoza, Mejía, Flores y Col. (2016) manifiestan que el problema de los embarazos en los adolescentes se presenta en toda Latinoamérica con altos índices de complicaciones durante la gestación, que conllevan un alto gasto socioeconómico para los estados en vías de desarrollo, en donde los sistemas de salud son precarios, y no se atiende de forma adecuada a este grupo prioritario.

En el estudio realizado por Cruz, Lastra y Lastre (2018)

El embarazo adolescente es una situación compleja en el que se encuentran un número de factores de riesgo relacionados con las pautas de crianza y el tipo de relaciones que los adolescentes construyen en su vida cotidiana, además de las escasas respuestas efectivas de las instituciones $y$, más directamente de los padres de estas mujeres (p. 7).

Por eso la necesidad de implementar políticas de salud pública a nivel local, regional y mundial que abarquen un trabajo multidisciplinario en beneficio del bienestar de este grupo poblacional con una participación de la sociedad y la familia.

La problemática del embarazo en Latinoamérica ha sido abordada más que como un problema de salud como un tema de valores, en el que confluyen factores psicológicos, culturales y sociales, la familia, las instituciones educativas y los medios de comunicación social. El contenido estudiado está relacionado con las condiciones socio ambientales y culturales que propician el embarazo adolescente y los riesgos de salud propios de esta etapa que se originan de las condiciones antes mencionadas. Por lo que, se planteó la pregunta de investigación ¿Cómo se ha abordado el tema del embarazo adolescente como un problema de salud pública en Latinoamérica? 


\section{Metodología}

\subsection{Tipo de Estudio}

La presente investigación corresponde a una revisión bibliográfica, que abordó el embarazo adolescente como un problema de salud pública en Latinoamérica con una investigación crítica.

\subsection{Estrategia de búsqueda}

La búsqueda de la información científica se desarrolló utilizando diferentes bases de datos digitales como: Medigraphic, Scielo, Redalyc, Latindex, BvSalud, Publindex, páginas web institucionales asociadas al área de la salud y libros analógicos. En las estrategias de búsqueda se utilizaron operadores boléanos: "prevalencia AND embarazos AND adolescentes, embarazos OR control prenatal"; "adolescencia AND embarazos OR problema biopsicosocial"; "cultura AND adolescentes embarazos OR Salud Publica".

\subsection{Selección de los estudios}

Como lo indica la Figura 1, se analizaron los estudios identificando su título, resumen y su lectura integral; los datos más relevantes se extrajeron a través de un exhaustivo análisis de los documentos, luego de la búsqueda y manejo de la organización de la información, utilizando el gestor bibliográfico Mendeley, siendo clasificados como relevantes, altamente relevantes e irrelevantes. Para la inclusión de los documentos en el trabajo se consideraron aquellos que cumplían con todos los criterios de elegibilidad, en total se encontraron 385 documentos, los cuales de acuerdo con los criterios de inclusión se seleccionaron 122, y tras el análisis de los títulos, resúmenes y artículo completo fueron utilizados para el desarrollo de introducción, así como, para los resultados y la discusión 26 artículos que fueron discutidos y ayudaron a responder la pregunta de investigación.

Figura 1

Flujograma de la búsqueda bibliográfica

\begin{tabular}{|c|c|c|c|}
\hline & $\begin{array}{l}\text { Documentos sin exclusión } \\
\qquad(\mathrm{n}=385)\end{array}$ & $\begin{array}{l}\text { Documentos potenciales } \\
\text { luego de excluirlos } \\
\text { (n=122) }\end{array}$ & $\begin{array}{c}\text { Documentos incluidos y } \\
\text { elegidos } \\
(n=26)\end{array}$ \\
\hline \multirow{7}{*}{$\begin{array}{c}\text { Documentos } \\
\text { digitales }\end{array}$} & $\begin{array}{l}\text { Redalyc } \\
(n=93)\end{array}$ & $\begin{array}{l}\text { Redalyc } \\
(n=23)\end{array}$ & $\begin{array}{c}\text { Redalyc } \\
(n=5)\end{array}$ \\
\hline & $\begin{array}{l}\text { Scielo } \\
(n=96)\end{array}$ & $\begin{array}{l}\text { Scielo } \\
(n=30)\end{array}$ & $\begin{array}{l}\text { Scielo } \\
(n=11)\end{array}$ \\
\hline & $\begin{array}{l}\text { Publindex } \\
(n=29)\end{array}$ & $\begin{array}{l}\text { Publindex } \\
(n=11)\end{array}$ & $\begin{array}{l}\text { Publindex } \\
(n=1)\end{array}$ \\
\hline & $\begin{array}{c}\text { BvSalud } \\
(n=21)\end{array}$ & $\begin{array}{c}\text { BvSalud } \\
(n=6)\end{array}$ & $\begin{array}{l}\text { BvSalud } \\
(n=1)\end{array}$ \\
\hline & $\begin{array}{c}\text { Medigraphic } \\
(n=27)\end{array}$ & $\begin{array}{c}\text { Medigraphic } \\
(n=5)\end{array}$ & $\begin{array}{l}\text { Medigraphic } \\
(n=2)\end{array}$ \\
\hline & $\begin{array}{c}\text { Latindex } \\
(n=86)\end{array}$ & $\begin{array}{c}\text { Latindex } \\
(n=28)\end{array}$ & $\begin{array}{l}\text { Latindex } \\
(n=1)\end{array}$ \\
\hline & $\begin{array}{l}\text { Páginas web institucionales } \\
\qquad(\mathrm{n}=29)\end{array}$ & $\begin{array}{l}\text { Páginas web institucionales } \\
\qquad(n=17)\end{array}$ & $\begin{array}{l}\text { Páginas web institucionales } \\
\qquad(\mathrm{n}=3)\end{array}$ \\
\hline $\begin{array}{l}\text { Documentos } \\
\text { analógicos }\end{array}$ & $\begin{array}{l}\text { Libros } \\
(n=4)\end{array}$ & $\begin{array}{l}\text { Libros } \\
(n=2)\end{array}$ & $\begin{array}{l}\text { Libros } \\
(n=2)\end{array}$ \\
\hline
\end{tabular}




\section{Resultados}

Una vez finalizada la búsqueda de la información científica se extrajeron 26 documentos científicos (100\%) de los cuales 15 documentos fueron utilizados para responder a la pregunta de investigación planteada, las investigaciones se seleccionaron de diferentes bases de datos, de las cuales algunas destacaron al presentar un mayor número de artículos que sirvieron para el desarrollo del trabajo (figura 2). Los estudios seleccionados presentaban metodologías de investigación de tipo prospectivo, observacional, experimental, cuantitativo, correlacional y analítico, además, se utilizaron ensayos clínicos de tipo cuasi experimental, prospectivo y multicéntricos.

Figura 2

Categorías de análisis

\section{Lineamientosteóricossobre el embarazo adolescente}

Establecer las tasas de embarazos adolescentes como problema de salud pública

Especificar losfactores determinantes del embarazo en las adolescentes

Identificar lascomplicaciones que enfrentan las adolescentes durante el embarazo

Fuente: elaboración propia

\subsection{Lineamientos teóricos del embarazo adolescente}

Autores como Papalia, Olds y Feldman (2009) definen a la adolescencia como "una transición del desarrollo, que implica cambios físicos, cognitivos, emocionales y sociales" (p. 461); además, manifiestan que entre los 11 y los 14 años los adolescentes crecen física, cognitiva y socialmente; sin embargo, es también una época de riesgos, pues hay adolescentes que no saben qué hacer con estos cambios y requieren de orientación; hay adolescentes que llegan a la madurez sin inconvenientes en el camino, pero, hay otros que se enfrentan a una serie de riesgos, como es su participación temprana en la actividad sexual y sin usar anticonceptivos, lo que suele traer como consecuencia un embarazo.

En la dimensión física de acuerdo con Gómez, Arruda, Luarte, Albornoz, Fierro, y Cossio (2016) se encuentra el "crecimiento somático (general), neural, genital y linfoide" (p.246), los cuales tienen relación directa con los factores genéticos y alimenticios; cuyo desarrollo puede afectar el comportamiento y la estabilidad emocional de los adolescentes.

En lo que se refiere a la dimensión cognitiva, Craig y Baucum (2009) “la mayoría de los adolescentes alcanzan el nivel del pensamiento operacional formal, que es abstracto, especulativo e independiente del entorno y las circunstancias inmediatas" (p. 372); los adolescentes se dan cuenta que tienen un presente, un pasado y un futuro; es cuando reconocen que todo acto tiene una consecuencia como cuando reflexionan si no uso el condón, lo más probable es que tenga un hijo o adquiera una enfermedad de transmisión sexual.

De acuerdo al Fascículo II de las Aldeas Infantiles SOS LAAM (2017) en el aspecto psicosocial, los adolescentes dejan de identificarse con sus padres o quienes estuvieron bajo su cuidado, se vuelven egocéntricos, piensan que 
son seres especiales que no requieren de supervisión; y, que tienen libertad de hacer con su sexualidad como ellos quieran sin ningún control, buscando información en los medios de comunicación que presentan una práctica de la actividad sexual sin límites, donde todo está permitido: desenfreno y violencia.

Flores, Nava y Arenas (2017) refieren que "el embarazo en la adolescencia es aquel que ocurre dentro de los dos años ginecológica" (p. 376); entendiéndose por tal al tiempo transcurrido desde la menarca y/o cuando la adolescente es aún dependiente de su núcleo familiar de origen. Continuando con Castillo (2016) menciona que "la OMS define que el embarazo en la adolescencia es el que ocurre en mujeres entre los 10 y 19 años, sin tener en cuenta otras consideraciones" (p. 546), esta concepción es muy reducida pues se limita a definirlo solo por la edad sin tomar en cuenta otros factores como educación, cultura, desarrollo físico y mental.

De acuerdo con los aportes de Barreto, Sáenz, Velandia y Gómez (2013) en estudio sobre embarazo adolescente

Es un intento de la adolescente de satisfacer su necesidad de ser reconocida, valorada y amada por su familia; un intento de emanciparse de un sistema familiar amalgamado que se trunca, generando más de lo mismo; o hace parte de un legado familiar con sus mitos y lealtades que no siempre ayuda al desarrollo de sus miembros (p. 848)

Convirtiéndose las adolescentes en presas de su propia inestabilidad emocional generada en su particular núcleo familiar reflejando síntomas de disfuncionalidad.

\subsection{Establecer las tasas de embarazos adolescentes como problema de salud pública}

Según Fajardo, Ramos, Padilla, Andrade, y Cuadra (2015) en estudio realizado en Honduras "un total de 14,008 nacimientos fueron atendidos en la Sala de Maternidad durante el año 2014, de los cuales 4,035 correspondían a madres adolescentes, con una prevalencia de $28,8 \%$ " (p.10), esto permite identificar que la prevalencia de embarazos adolescentes en este hospital es alta, reflejando una problemática social que requiere de atención de las autoridades de la salud.

Por su parte Díaz, Velasco, Gutiérrez, Muro, y Álvarez (2019)

Muestra la incidencia del embarazo en edades tempranas en los años 2015, 2016 y 2017, donde su frecuencia se comportó de manera similar en todos los años. Al evaluar la distribución de gestantes según edad predominó el grupo de edades de 17 - 19 años con 143 casos (80,8 \%), seguido del grupo de 14 16 años con 34 casos $(19,2 \%)$ (p.7).

Una realidad muy cercana a todos los países latinoamericanos que refleja la falta de conciencia por parte de las adolescentes en el momento de iniciar su vida sexual sin ningún tipo de control o método de anticoncepción, convirtiéndose en una problemática de salud que influye de manera negativa en los índices de salud de una nación.

Ecuador tiene la tasa más alta de embarazo en adolescentes a nivel de América Latina y El Caribe, según el último informe del Fondo de Población de las Naciones Unidas (UNFPA), denominado "El poder de decidir. Los derechos reproductivos y la transición demográfica" (2018):

111 de cada mil jóvenes entre 15 y 19 años han estado embarazadas; le sigue en el índice Honduras (103), Venezuela (95), Nicaragua (92) y Guatemala (92) con las mayores tasas. Y los países de la región con una menor tasa son Chile (41), Trinidad y Tobago (38), Curaçao (35) (p.132).

Vinueza y Bello (2018), en su investigación realizada en una clínica particular de Ecuador en el año 2017 sobre embarazo en la adolescencia concluyen que "predominaron en la población estudio las gestantes adolescentes solteras con parto anterior. La no utilización de algún método anticonceptivo y los antecedentes de infecciones 
asociadas al embarazo prevalecen en este grupo" (р.319), lo que confirma que se hace necesario implementar programas en salud sexual y reproductiva a nivel del grupo poblacional adolescente para que reflexionen sobre la importancia de prevenir un embarazo no deseado mediante el uso de anticonceptivos adecuados.

\subsection{Especificar los factores determinantes del embarazo en las adolescentes}

Castañeda, De León, y Brazil (2019) en su investigación realizada en el contexto binacional México-Estados Unidos concluyen "los factores asociados al embarazo y la maternidad temprana, se relacionan con un conjunto de condiciones socioestructurales y sociosimbólicas que imperan en la familia, la escuela y la comunidad" (p.327), convirtiéndose la triada familia, escuela y comunidad en los espacios idóneos para ejercer prácticas de exclusión y desigualdad de género, siendo las adolescentes sujetos vulnerables a la discriminación y violencia.

Quintero (2016) establece que "el embarazo en estas edades mayoritariamente es no deseado y el aborto como vía de finalizarlo constituye también un problema de salud que se presenta con gran frecuencia" (p. 268), convirtiéndose en una problemática biopsicosocial que confirma la necesidad de implementar políticas gubernamentales para prevenir los embarazos no deseados en las adolescentes y evitar complicaciones médicas.

Autores como Sánchez et al. (2019) mencionan que "en Ecuador el embarazo en la adolescencia es considerado por parte de las autoridades sanitarias, como un problema de salud, dada sus consecuencias sociales y de salud de las gestantes" (p. 3), constituyéndose en grupos de riesgos al presentar complicaciones obstétricas, generando mayor gasto económico al Estado en la atención de salud.

\subsection{Identificar las complicaciones que enfrentan las adolescentes durante el embarazo}

Mora y Hernández (2015) en su investigación sobre embarazo en la adolescencia mencionan que

En México el $20 \%$ de los nacimientos anuales ocurren en mujeres menores de 20 años. La importancia de esto radica en que la repercusión del embarazo en las adolescentes empieza con los riesgos para la salud de la madre y su hijo (p. 294).

Estos riesgos pueden ser aborto, anemia, infección en las vías urinarias, hipertensión arterial y parto prematuro en la madre y, en el caso del hijo, bajo peso al nacer, problemas neurológicos y riesgo de muerte en el primer año de vida.

En la investigación de Rodríguez, Cala, Nápoles, Milán, y Aguilar (2017) en Cuba "resultaron factores de riesgo para el embarazo en la adolescencia el tener sus primeras relaciones sexuales entre los 15 y 19 años y no poseer conocimiento sobre complicaciones del embarazo en la adolescencia" (p.29), lo que indica que la educación sexual a los adolescentes dentro de las familias y en las escuelas debe estar dirigida a la implementación de métodos anticonceptivos y, de esta manera, prevenir los embarazos adolescentes.

Castillo (2016) en su investigación realizada en Colombia

El 20\% de las mujeres embarazadas son adolescentes, aunque la mortalidad materna es de 2 a 5 veces más alta en este ciclo vital que entre las mujeres de 20 a 29 años. Además de las complicaciones materno perinatales asociadas con la inmadurez biológica, tener un hijo antes de los 20 años genera un impacto socioeconómico negativo que puede prolongarse por toda la vida (p.544).

Lo que permite identificar que las consecuencias de un embarazo adolescente no solo implican problemas de salud tanto para la madre como para el hijo, también entraña dificultades a nivel económico que suele ser el común denominador en la Región Latinoamericana. 
Okumura, Maticorena, Tejeda, y Mayta (2014) en su estudio realizado en Lima encontraron que existe "mayor riesgo de cesárea e infección puerperal en las adolescentes menores de 15 años, así como mayor riesgo de episiotomía en las adolescentes tardías" (p. 383), lo que demuestra la vulnerabilidad de las adolescentes en lo que se refiere a las complicaciones obstétricas y perinatales, tales como preclamsia, hemorragia y parto pretérmino.

\section{Conclusiones}

La adolescencia es una etapa de transición de la niñez a la adultez, en la que los sujetos sufren cambios a nivel físico, cognitivo y psicosocial. A los adolescentes se les hace difícil manejar estos cambios y esta es la razón por la que buscan salidas de lo más variadas: anorexia, bulimia, consumo y abuso de alcohol y drogas, relaciones sexuales sin utilizar condones que pueden provocar enfermedades de transmisión sexual o un embarazo. El embarazo adolescente es una realidad de la familia latinoamericana, tan venida a menos en la actualidad; que se ve reemplazada por un mundo virtual y tecnológico, en el que los adolescentes encuentran respuestas a sus demandas, dejando a un lado la autoridad de los padres o de sus cuidadores principales.

Ecuador tiene la tasa más alta de embarazo adolescente en Latinoamérica y El Caribe, seguido de países como Honduras y Venezuela, la edad predominante de mujeres adolescentes embarazadas lo constituye entre 17 a 19 años de edad, sin embargo, se presentan también embarazos en edades que van entre los 14 y 16 años, vislumbrando una problemática que requiere atención desde las instancias de la salud. Sin embargo, las tasas son inversamente proporcionales; a mayor calidad de servicios, educación y cultura será menor el número de embarazos, convirtiéndose a esta población en un grupo etario vulnerable por sus características psicológicas y sociales.

En función de estos datos, se concluye que en Latinoamérica el número de embarazos adolescentes es creciente, lo cual indica que algo está fallando en la educación sobre el manejo de la sexualidad a temprana edad. Esta es la razón por la que se hace necesario que los estados revisen sus políticas de salud pública en cuanto a la prevención de embarazos adolescentes y adoptar programas de educación en salud que involucren a los adolescentes, la familia, la escuela y la comunidad.

El embarazo adolescente está relacionado con factores socio culturales y socio económicos, dependiendo del modelo de crianza establecido en cada familia. Asimismo, la escuela y la comunidad desempeñan un rol determinante puesto que la sociedad latinoamericana en la actualidad todavía vulnera y excluye a las adolescentes embarazadas; pese a existir leyes y normativas que amparan a este grupo poblacional.

Las complicaciones durante el parto son más frecuentes mientras más temprano sea el embarazo, debido a que no alcanza la debida madurez física y psicológica de la mujer; por ende, se incrementa la mortalidad materna y perinatal. En el caso de la madre, las complicaciones son: eclampsia, hipertensión gestacional, hemorragia posparto; en cuanto al recién nacido son: dificultad respiratoria, sepsis bacteriana del recién nacido, otras malformaciones congénitas del corazón. En lo que se refiere a las complicaciones psicológicas de la madre se presenta: abandono de los estudios, mayor riesgo de desarrollar depresión y suicidio, lo que le lleva a la obtención de empleos con bajo sueldo que repercute en vivir en condiciones de pobreza, pues, en muchos casos no tienen una pareja que le apoye económicamente, determinando un núcleo familiar disfuncional que tiende a perpetuar la problemática social a nivel transgeneracional. 


\section{Referencias bibliográficas}

Aldeas Infantiles SOS LAAM. (2017). Desarrollo biopsciosocial en la adolescencia y juventud: Una aproximación desde la integralidad para la promoción del bienestar socioemocional. Recuperado de https://www.aldeasinfantiles.org/getmedia/46fe8124-38f4-42d3-a391-d2039ab78b3c/Fasciculo-IIDesarrollo-Biopsicosocial-en-la-Adolescencia-y-Juventud.pdf

Barreto, E., Sáenz, M., Velandia, F. y Gómez, J. (2013) Embarazo en adolescentes bogotanas: Significado relacional en el sistema familiar. Revista de Salud Pública, 15(6), 837-849. Recuperado de https://revistas.unal.edu.co/index.php/revsaludpublica/article/view/38736/46274

Castañeda, N., De León, M. y Brazil, L. (2019). Embarazo y maternidad: percepciones de las jóvenes en un contexto binacional México-Estados Unidos. Revista Latinoamericana de Ciencias Sociales, Niñez y Juventud, 17 (1), 327-342. Recuperado de http://www.scielo.org.co/pdf/rlcs/v17n1/2027-7679-rlcs-17-0100327.pdf

Castillo, L. (2016). Desaciertos en la prevención del embarazo en adolescentes. Revista Salud Uninorte, 32(3), 543-551. Recuperado de http://www.scielo.org.co/pdf/sun/v32n3/v32n3a16.pdf

Craig, G. y Baucum, D. (2009). Desarrollo psicológico (9a ed.). México: Pearson Educación.

Cruz, M., Lastra, N. y Lastre, G. (2018). Revisión de conceptos: Embarazo en adolescentes y la experiencia en el control prenatal. Ciencia de Innovación en Salud, 6(2), 1-21. Recuperado de http://revistas.unisimon.edu.co/index.php/innovacionsalud/article/view/3100

Díaz, A., Velasco, A., Gutiérrez, J. Muro, J. y Álvarez J. (2019). Embarazo en la adolescencia, un problema de salud en el policlínico "Efraín Mayor Amaro" del Cotorro, La Habana. Revista Cubana de Obstetricia y Ginecología, 45(2), 1-15. Recuperado de http://revginecobstetricia.sld.cu/index.php/gin/article/view/450/379

Fajardo, I., Ramos, G., Padilla, J., Andrade, S. y Cuadra, Y. (2015). Prevalencia de embarazo en adolescentes, Hospital Leonardo Martínez Valenzuela. Revista Científica Universidad, 2(2). 10-20. Recuperado de http://www.bvs.hn/RCEUCS/pdf/RCEUCS2-2-2015-4.pdf

Fawed, O., Erazo, A., Carrasco, J.C., Gonzáles, D., Mendoza, A. F., Mejía, M. E., Flores, J. C, y otros. Complicaciones Obstétricas en Adolescentes y Mujeres Adultas con o sin Factores de Riesgo Asociados, Honduras 2016. iMedPub Journals, 12(4), 1-7. Recuperado de https://www.archivosdemedicina.com/medicina-de-familia/complicaciones-obsteacutetricas-enadolescentes-y-mujeres-adultas-con-o-sin-factores-de-riesgo-asociados-honduras-2016.pdf

Flores, M., Nava, G. y Arenas, L. (2017). Embarazo en la adolescencia en una región de México: un problema de Salud Pública. Revista de Salud Pública, 19(3), 374-378. Recuperado de https://www.redalyc.org/articulo.oa?id=422/42254536013

Fondo de Población de las Naciones Unidas. (2018). Estado de la población mundial 2018. El poder de decidir: Derechos reproductivos y transición demográfica. Recuperado de https://www.unfpa.org/sites/default/files/pubpdf/UNFPA_PUB_2018_ES_SWP_Estado_de_la_Poblacion_Mundial.pdf 
Gaete, V. (2015). Desarrollo psicosocial del adolescente. Revista chilena de pediatría, 86(6), 436-443. Recuperado de https://scielo.conicyt.cl/pdf/rcp/v86n6/art10.pdf

Gómez, R., Arruda, M., Luarte, C., Albornoz, C., Firerro, A., y Cossio, M.(2016). Enfoque teórico del crecimiento físico de niños y adolescentes. Revista Española de Nutrición Humana y Dietética, 20(3), 244-253. Recuperado de http://scielo.isciii.es/scielo.php?script=sci_arttext\&pid=\$2174-51452016000300011

Loredo, A., Vargas, E., Casas, A., González, J. y Gutiérrez, C. (2017). Embarazo adolescente: su causas y repercusiones en la diada. Revista Médica del Instituto Mexicano del Seguro Social, 55(2), 224-229. Recuperado de https://www.redalyc.org/jatsRepo/4577/457750722016/index.html

Molina, A., Pena, R., Díaz, C., Soto, M. (2019). Condicionantes y consecuencias sociales del embarazo en la adolescencia. Revista Cubana de Obstetricia y Ginecología, 45(2), e218. Recuperado de http://scielo.sld.cu/scielo.php?script=sci_arttext\&pid=S0138$600 \times 2019000200017 \& \operatorname{lng}=e s \& n r m=i s o \&$ tlng=es

Mora, A., y Hernández, M. (2015). Embarazo en la adolescencia. ¿Qué representa para la sociedad actual? Revista Ginecología y Obstetricia de México, 83(5), 294-301. Recuperado de https://www.medigraphic.com/pdfs/ginobsmex/gom-2015/gom155e.pdf

Morín, A., Treviño, M. y Rivera, G. (2019). Embarazo en estudiantes universitarias. Revista Médica del Instituto Mexicano del Seguro Social, 57(1), 4-5. Recuperado de https://www.redalyc.org/jatsRepo/4577/457759795004/457759795004.pdf

Okumura, J., Maticorena, D., Tejeda, J. y Mayta, P. (2014). Embarazo adolescente como factor de riesgo para complicaciones obstétricas y perinatales en Lima-Perú. Revista Brasileira de Saúde Materno Infantil, 14(4), 383-392. Recuperado de http://www.scielo.br/scielo.php?script=sci_arttext\&pid=S151938292014000400383

Organización Mundial de la Salud. (2018). Aceleración mundial de las medidas para promover la salud de los adolescentes (Guía AA-HA!): Orientación para la aplicación en los países. Whashington, D.C.: Organización Panamericana de la Salud. Recuperado de https://iris.paho.org/handle/10665.2/49181

Papalia, D., Olds S. y Feldman, R. (2009). Psicología del desarrollo. De la infancia a la adolescencia. (11aㅡ ed.). México: McGrawHill Educación.

Pinzón, A.M., Ruiz, A. M., Aguilera, P. A., y Abril, P. S. (2018). Factores asociados al inicio de vida sexual y al embarazo adolescente en Colombia. Estudio de corte transversal. Revista chilena de obstetricia y ginecología, 83(5), 487-499. Recuperado de https://scielo.conicyt.cl/scielo.php?pid=S0717$75262018000500487 \&$ script=sci_arttext\&tlng=p

Quintero, P. (2016). Caracterización de los factores de riesgo del embarazo en la adolescencia en el Policlínico Universitario "Pedro Borrás Astorga”. Revista Cubana de Obstetricia y Ginecología, 42(3), 262-273. Recuperado de https://www.medigraphic.com/pdfs/revcubobsgin/cog-2016/cog163b.pdf

Rodríguez, N., Cala, A., Nápoles, J., Milán, Y. y Aguilar, M. (2017). Factores de riesgo asociados al embarazo en adolescentes. Revista Información Científica, 96(1), 29-37. Recuperado de http://www.revinfcientifica.sld.cu/index.php/ric/article/view/27/780 
Sánchez, S., Bastidas, G., Alfonso, I., y Labrada, E. (2019). Embarazo no deseado y sus factores asociados en estudiantes universitarias. Revista Espacios, 40(39), 1-11. Recuperado de https://www.revistaespacios.com/a19v40n39/a19v40n39p09.pdf

Vinueza, M., y Bello, H. (2018). Embarazo en la adolescencia: Características epidemiológicas. Revista Científica Dominio de las Ciencias, 4(1), 311-321. Recuperado de file://C:/Users/ZONA\%2OINFORMATICA/Downloads/DialnetEmbarazoEnLaAdolescenciaCaracteristicasEpidemiolog-6313257.pdf

Esta obra está bajo una Licencia Creative Commons Attribución-NoCommercial 4.0 International

\section{(c) $)$ BY-NC}

